\title{
ADVANCED OXIDATION PROCESS (AOPS) FOR MICROPOLLUTANT TREATMENT: A REVIEW
}

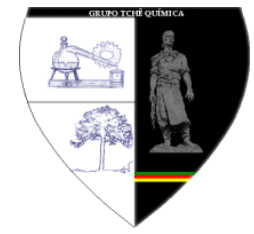

\author{
KUFFEL, Fernando J. M. ${ }^{1}$; BRANDT, Cassiano R. ${ }^{1}$; KUHN, Daniel ${ }^{1}$; STEFFENS, Clarice ${ }^{2}$; STULP, \\ Simone ${ }^{1}$; ECKHARDT, Rafael R. ${ }^{1}$; REISDÖRFER, Gustavo ${ }^{1}$; SILVA, Maria C. A. ${ }^{1}$; HILGEMANN, \\ Maurício $^{1}$; HOEHNE, Lucélia ${ }^{1^{*}}$ \\ ${ }^{1}$ Centro Universitário Univates, Rua Avelino Tallini, 171, cep 95900-000, Lajeado - RS, Brasil \\ (fone: (51) 3714-7000; Ramal: 5524) \\ ${ }^{2}$ Universidade Regional Integrada do Alto Taquari e das Missões, Av. Sete de Setembro, 1621, cep 99709-910, \\ Erechim - RS, Brasil \\ (fone: (54) 3520-9000) \\ * Lucélia Hoehne \\ e-mail: luceliah@univates.br
}

Received 16 October 2016; received in revised form 03 November 2016; accepted 29 November 2016

\section{RESUMO}

Com a crescente preocupação na qualidade da água potável disponível no planeta, uma nova classe de poluentes emergentes em rios e lagos tem chamado atenção da comunidade científica: os micropoluentes. Tais compostos provém do despejo irregular ou involuntário de substâncias tais como pesticidas, fármacos, inseticidas e entre outros na natureza. Por possuírem uma estrutura complexa, os micropoluentes possuem alta resistência aos métodos tradicionais de tratamento de água para consumo humano. Deste modo, têm se estudado metodologias alternativas para sua degradação, tal como os processos oxidativos avançados (POAs). Os POAs baseiam-se na formação de radicais hidroxilas $\left(\mathrm{OH}^{-}\right)$altamente reativos que degradam substâncias complexas em produtos finais menos agressivos, tais como $\mathrm{CO}_{2}$ e água. Apesar de existirem uma série de métodos para a geração do radical hidroxila a partir de processos oxidativos avançados, estas diferenciam quanto a aplicação e viabilidade. Nesta revisão são apresentadas as diferentes metodologias empregadas no uso de POAs e suas respectivas características de aplicação, bem como alguns trabalhos já realizados no tratamento de substâncias complexas que comprovando sua eficiência.

Palavras-chave: fármacos; tratamento de água; poluição; Oxidação; luz Ultra-Violeta.

\begin{abstract}
The growing preoccupation about drinking water quality available on earth, a new order of emergent pollutants in rivers and lakes has called the attention of the scientific community, the micropollutants. These compounds come from the irregular or involuntary ejectment of substances such as pesticides, drugs, insecticides and other into the nature. By having a complex structure, the micropollutants have high resistance over the traditional water treatment to human consume. Thus, alternatives methodologies to their degradation have been studied, such as advanced oxidation process (AOPs). The AOPs are based in the formation of hydroxyls radicals $\left(\mathrm{OH}^{-}\right)$highly reactive that degrade complexes substances into lesser aggressive products as $\mathrm{CO}_{2}$ and water. Even though many methods to generate hydroxyls radicals from advanced oxidation processes exist, they differentiate on the application and viability. This review shows different methodologies using AOPs and their respective application characteristics, also some works already made in complexes substances treatment, which prove their efficiency.
\end{abstract}

Keywords: drugs; water treatment; pollution; oxidation; ultraviolet light.

PERIÓDICO TCHÊ QUÍMICA • www.periodico.tchequimica.com • Vol. 14 N. 27. • ISSN 1806-0374 (impresso) • ISSN 1806-9827 (CD-ROM) • ISSN 2179-0302 (meio eletrônico) 
INTRODUÇÃO

A preocupação com a qualidade de água para consumo existe a séculos, contudo, com o passar do tempo e através das mudanças de hábito do ser humano, o tipo de contaminação nos rios e efluentes mudou (Bolong, Ismail, Salim 2009). Sendo assim, uma nova ordem de contaminantes têm chamado atenção da comunidade científica, o que está acarretando em diversos estudos na área (Liu et al. 2014).

Os chamados micropoluentes emergentes, por terem uma estrutura complexa, causam uma grande preocupação devido a sua alta resistência aos métodos tradicionais utilizados para tratamento de água potável (Wols, Hofman-Caris 2012). Agregados a essa ordem de poluentes se encontram antibióticos, produtos de cuidado e higiene pessoal, inseticidas e pesticidas entre outros (Pal et al. 2010, Gros, Petrovic, Barceló 2008).

Estudos realizados comprovam que, mesmo estando em baixas concentrações, a presença de tais poluentes em cursos de água pode causar danos aos seres humanos e à fauna marinha em médio e longo prazo (Bila, Dezotti 2007; Snyder et al. 2003,). Frente a tais problemas, um novo método para o tratamento eficaz e viável dos micropoluentes, conhecido como Processos Oxidativos avançados (POAs), tem sido estudado e aprimorado (Thepsithar e Roberts 2006; Quan et al. 2005; Wang, Jiang, Liu 2008).

Os POAs são caracterizados pela capacidade de produzir radicais hidroxila $(\mathrm{OH})$ altamente reativos $\left(\mathrm{E}^{\circ}=2,80 \mathrm{eV}\right)$, promovendo assim a degradação de vários compostos de difícil degradação (Cheng et al. 2016; Rodríguez et al. 2005). O interesse nos POAs não é recente, contudo, apenas atualmente, com a crescente quantidade de tais poluentes presentes nos cursos de água, tem se intensificado o estudo dos diversos tipos de processos de oxidação (Ganiyu et al. 2015).

Os radicais hidroxila são formados a partir de oxidantes como peróxido de hidrogênio $\left(\mathrm{H}_{2} \mathrm{O}_{2}\right)$ ou gás ozônio $\left(\mathrm{O}_{3}\right)$, e sua eficiência pode ser aumentada através da irradiação ultravioleta (UV) ou catalisadores. A radiação UV por sua vez, reage diretamente com as moléculas de água, $\mathrm{H}_{2} \mathrm{O}_{2}$ ou $\mathrm{O}_{3}$ e forma o radical $\mathrm{OH}$, o qual oxida a matéria presente no meio. $O$ produto final da reação gera compostos não agressivos, tais como $\mathrm{CO}_{2}$ e $\mathrm{H}_{2} \mathrm{O}$ (Ikehata, Naghashkar, El-Din
2006).

Os POAs compreendem uma série de métodos para a geração do radical $\mathrm{OH}$, sendo alguns deles: Fenton, foto-fenton, foto catálise, ozonização e UV/ $\mathrm{H}_{2} \mathrm{O}_{2}$ (Comninellis et al. 2008).

A melhoria no tratamento de efluentes industriais também tem sido constantemente estudada. No trabalho de Rodrigues et al. (2010), desenvolveu-se um reator de eletrofloculação com eletrodo de alumínio para o tratamento de água em uma plataforma de petróleo. $O$ estudo, focado na remoção de óleos e graxas, cor, turbidez, apresentou resultados de, em média, $80 \%$ na remoção destes.

\section{METODOLOGIAS}

\subsection{Fenton}

O processo Fenton tem por base o uso de ferro para formação da hidroxila (Ay, Kargi 2010). $\mathrm{O}$ íon de ferro $\left(\mathrm{Fe}^{2+}\right)$ reage com $\mathrm{O} \mathrm{H}_{2} \mathrm{O}_{2}$ segundo a equação 1 (Chen, Juan e Wei 2005).

$$
\mathrm{Fe}^{2+}+\mathrm{H}_{2} \mathrm{O}_{2} \rightarrow \mathrm{Fe}^{3+}+\mathrm{OH}^{-}+\mathrm{OH}^{\cdot}
$$

$\mathrm{O} \mathrm{pH}$ ideal para o acontecimento da reação é em torno de 3 , pois quando o $\mathrm{pH}$ se encontra abaixo desse valor $\circ \mathrm{H}_{2} \mathrm{O}_{2}$ pode tornarse um íon instável $\left(\mathrm{H}_{3} \mathrm{O}_{2}{ }^{+}\right)$, o que reduz sua reatividade com o íon ferro. A concentração de peróxido de hidrogênio também pode variar conforme a quantidade de ferro utilizada (Kang, Hua e Rao, 2006). O processo de fenton pode ser realizado a uma temperatura ambiente (23-25 $\left.{ }^{\circ} \mathrm{C}\right)$ (Cheng et al. 2016; Hsueh et al. 2005).

Este processo é muito atrativo e usado pois o ferro é um material economicamente viável, abundante e não tóxico. Em condições laboratoriais a reação de Fenton tem se mostrado capaz de degradar moléculas orgânicas e inorgânicas (Ayoub et al. 2010).

\subsection{Foto-Fenton}

O processo foto-Fenton assemelha-se ao processo de Fenton devido ao uso de ferro, contudo, o processo de foto-fenton difere-se devido ao uso de luz UV-visível, o que favorece a oxidação dos poluentes com a luz de irradiação apresentando um comprimento de onda $>300 \mathrm{~nm}$ (Neyens, Baeyens 2003).

$\mathrm{O} \mathrm{pH}$ nesse processo deve estritamente controlado e estabilizado em torno de 2.8 , onde

PERIÓDICO TCHÊ QUÍMICA • www.periodico.tchequimica.com • Vol. 14 N. 27 - ISSN 1806-0374 (impresso) • ISSN 1806-9827 (CD-ROM) • ISSN 2179-0302 (meio eletrônico) 
metade do $\mathrm{Fe}$ (III) está presente como íon $\mathrm{Fe}^{3+} \mathrm{e}$ a outra metade como íon $\mathrm{Fe}(\mathrm{OH})_{2}{ }^{+}$(Lázár et al. 2004). Quando o pH está acima de 2,8, o Fe(III), precipitará como oxihidróxido de ferro. Já quando o pH está abaixo de 2,8, a concentração do íon $\mathrm{Fe}(\mathrm{OH})_{2}{ }^{+}$decairá (Cheng et al. 2016).

\subsection{Foto-Catálise}

A foto catálise utiliza-se de semicondutores de óxido de metais, tais como $\mathrm{TiO}_{2}, \mathrm{ZnO}, \mathrm{CdS}, \mathrm{GaP}, \mathrm{WO}_{3}$, entre outros. Dentre eles o mais utilizado é $\mathrm{o} \mathrm{TiO}_{2}$, sendo tal preferência devido a sua alta foto reatividade, inércia química, não toxicidade, baixo custo e fácil obtenção (Ganiyu et al. 2015).

Sendo um foto-catalisador e respeitando as características de um semicondutor, $0 \quad \mathrm{TiO}_{2}$ deve receber energia para que haja a movimentação de elétrons entre as bandas gaps de valência para de condução, gerando assim o par elétron-lacuna, segundo equação 2 (Ohtani 2014; Cheng et al. 2016). A partir disto, as lacunas $\left(\mathrm{h}^{+}\right)$podem gerar radicais hidroxilas, equação 3 , ou reagirem com compostos adsorvidos, equação 4 , enquanto o elétron $\left(\mathrm{e}^{-}\right)$ por sua vez reduz o oxigênio dissolvido em superóxidos e posteriormente em hidroxilas, equação 5 (Ribeiro et al. 2015).

$$
\begin{aligned}
& \mathrm{TiO}_{2}+h v \rightarrow e^{-}+h^{+} \\
& \mathrm{TiO}_{2}\left(h^{+}\right)+\mathrm{H}_{2} \mathrm{O} \rightarrow \mathrm{TiO}_{2}+\mathrm{OH}^{\cdot}+H^{+} \\
& \mathrm{TiO}_{2}\left(h^{+}\right)+R X_{a d} \rightarrow \mathrm{TiO}_{2}+\mathrm{RX}_{a d}^{\cdot+} \\
& \mathrm{TiO}_{2}\left(e^{-}\right)+\mathrm{O}_{2} \rightarrow \mathrm{O}_{2}^{\cdot-}
\end{aligned}
$$

Uma das vantagens do $\mathrm{TiO}_{2}$ é que a energia necessária para a excitação dos elétrons corresponde a uma luz UV com comprimento de onda $<387 \mathrm{~nm}$, o que permite o uso da luz solar natural (Giraldo-Aguirre et al. 2015; Serna-Galvis et al. 2016).

Outro semicondutor que vem ganhando atenção de pesquisas é o $\mathrm{ZnO}$. Este possui características foto-oxidativas similares ao $\mathrm{TiO}_{2}$, com a vantagem de ser um melhor condutor de elétrons. Entretanto, pelo fato do $\mathrm{ZnO}$ ser mais corrosivo, $\mathrm{O} \mathrm{TiO}_{2}$ ainda é o mais indicado para a foto-catálise (Fenoll et al. 2013).

\subsection{Ozonização}

O método de ozonização, por ser pouco ofensivo ao meio ambiente, é largamente utilizado para fim de remoção de poluentes em efluentes. Por ter um potencial de redução alto $\left(E^{\circ}=2,07 \mathrm{~V}\right)$ é considerado um forte agente oxidante. Há duas formas de se utilizar o ozônio para a oxidação, a forma direta e a indireta (von Gunten 2003; Alvárez et al. 2006).

$\mathrm{Na}$ Tabela 1, pode ser visto a potência padrão de redução de radicais e compostos usando diferentes espécies.

Tabela 1: Potência Padrão de Redução de radicais e compostos

\begin{tabular}{c|c}
\hline Espécie & $\begin{array}{c}\text { Potência de } \\
\text { Redução (V) }\end{array}$ \\
\hline Flúor & $+3,03$ \\
Radical Hidroxila & $+2,80$ \\
Oxigênio Atômico & $+2,42$ \\
Ozônio & $+2,07$ \\
Peróxido de Hidrogênio & $+1,78$ \\
Permanganato & $+1,68$ \\
Dióxido de Cloro & $+1,57$ \\
Cloro & $+1,36$ \\
lodo & $+0,54$ \\
\hline
\end{tabular}

Fonte: dos Autores

$\mathrm{Na}$ forma direta a molécula de ozônio $\left(\mathrm{O}_{3}\right)$, ataca as ligações insaturadas dos compostos, quebrando a sua estrutura, contudo, apenas algumas partes do composto original são degradadas. Já na forma indireta, onde a eficiência é maior em $\mathrm{pH}$ alto, hidroxilas reagem com a matéria orgânica (Parrino et al. 2015). Para maior rendimento do ozônio adiciona-se $\mathrm{H}_{2} \mathrm{O}_{2}$, luz UV, ou catalisador sólido (Asghar, Raman, Daud 2015). Com $\mathrm{O} \quad \mathrm{H}_{2} \mathrm{O}_{2}$ a decomposição do $\mathrm{O}_{3}$ em radicais hidroxila aumenta conforme a equação 6 (Hansen et al. 2016)

$$
\mathrm{H}_{2} \mathrm{O}_{2}+2 \mathrm{O}_{2} \rightarrow 2 \mathrm{OH}^{\circ}+3 \mathrm{O}_{2}
$$

O ozônio pode reagir com os alcanos e romper suas ligações $\mathrm{C}-\mathrm{H}$ (Fokin e Schreiner 2002).

\section{$2.5 \mathrm{H}_{2} \mathrm{O}_{2} / \mathrm{UV}$}


$\mathrm{O}$ tratamento $\mathrm{H}_{2} \mathrm{O}_{2} / \mathrm{UV}$ acontece pela ação da irradiação de lâmpadas UV com comprimento abaixo de $400 \mathrm{~nm}$, que fotolisa as moléculas de $\mathrm{H}_{2} \mathrm{O}_{2}$ em dois radicais hidroxila, como mostra a equação 7 (Liu et al. 2015).

$$
\mathrm{H}_{2} \mathrm{O}_{2}+h v \rightarrow 2 \mathrm{OH}^{\cdot}
$$

As lâmpadas mais utilizadas são de vapor de mercúrio de baixa e média pressão, sendo sua emissão máxima de $254 \mathrm{~nm}$, pois $\mathrm{o} \mathrm{H}_{2} \mathrm{O}_{2}$ absorve no máximo 220 nm (Lopez et al. 2003).

Um ponto que deve ser considerado e se dar atenção é com a concentração de peróxido utilizado. Com uma concentração muito baixa, não há oxidação suficiente. Já com uma concentração muito elevada, há formação de peróxido residual (Pereira, Linden, Weinberg 2007; Lutterbeck et al. 2015). A concentração utilizada depende da aplicação e da quantidade de resíduo a ser degradado (Hyun Jo, Dietrich e Tanko 2011). Para eliminar o peróxido residual, alguns estudos trazem como eficiente a utilização controlada da enzima Catalase ou Bissulfato de Sódio $\left(\mathrm{NaHSO}_{4}\right.$ ) (Yuan et al. 2011; Giannakis et al. 2015; Vilhunen et al. 2010).

Este é um método muito estudado para se aplicar na degradação de resíduos de fármacos e desreguladores endócrinos (Benotti et al. 2009; Linden et al. 2007; Snyder, Wert e Lei 2007).

\section{DISCUSSÃO}

Segundo Giannakis et al. (2015), Yang et al. (2016) e Santos (2015), é possível utilizar POAs para o tratamento de efluentes de estação de tratamento que contenham micropoluentes. Pode-se verificar que Giannakis obteve resultados satisfatórios utilizando uma lâmpada de mercúrio de $9 \mathrm{~W}$ com comprimento de onda de $254 \mathrm{~nm}$. As amostras foram irradiadas por 10 e 30 minutos respectivamente, combinadas com $25 \mathrm{mg} / \mathrm{L}$ de peróxido de hidrogênio. O peróxido residual foi neutralizado com bissulfato de sódio $\left(\mathrm{NaHSO}_{4}\right)$. Depois de 10 minutos de irradiação, $100 \%$ dos micropoluentes foram removidos.

Para o melhor desempenho dos POAs na oxidação de compostos tem se desenvolvido diversos tipos de reatores. Tais mecanismos podem variar quanto às dimensões, formato, modelo e número de lâmpadas, concentração de $\mathrm{H}_{2} \mathrm{O}_{2}$, e concentração de ferro (quando utilizado).

Alguns autores, tal como Santos,
Meireles, Lange (2015), utilizaram um reator de foto degradação cilíndrico com as dimensões de $50,5 \times 320 \mathrm{~mm}$ e capacidade de $280 \mathrm{~mL}$, com um tubo de quartzo para proteger a lâmpada. A lâmpada utilizada foi de vapor de mercúrio de baixa pressão $(6 \mathrm{~W})$, emitindo radiação de 254 $\mathrm{nm}$ de comprimento de onda. Todos os experimentos forma feitos sob temperatura ambiente. Para os experimentos, houve variação da concentração de peróxido de hidrogênio, de 0,7 a $4,2 \mathrm{mmol} / \mathrm{L}$ e um tempo de reação de 100 minutos. O composto tratado experimentalmente foi o antibiótico Norfloxacina.

Por sua vez, Wols et al. (2015) traz em seu estudo dois modelos de reatores baseados na utilização do método de oxidação avançada usando $\mathrm{UV} / \mathrm{H}_{2} \mathrm{O}_{2}$. Ambos os modelos utilizaram os mesmos padrões de amostra, vazão, concentração de peróxido de hidrogênio e lâmpada. Contudo, houve uma variação nas dimensões dos reatores e na quantidade de lâmpadas utilizadas. Para o projeto utilizou-se uma lâmpada de mercúrio de baixa pressão de $120 \mathrm{~W}$ de potência, com comprimento de onda de $254 \mathrm{~nm}$. Além disso, a vazão dos fármacos foi fixada em $10 \mathrm{~L} / \mathrm{h}$ com uma concentração entre 1 e $5 \mu \mathrm{m}$. Já a vazão de peróxido de hidrogênio, com uma concentração de $5 \mathrm{~g} / \mathrm{L}$, foi mantida a 2 $\mathrm{L} / \mathrm{h}$. Os reatores foram construídos em formato cilíndrico, sendo o primeiro modelo com 1050 $\mathrm{mm}$ de comprimento por $130 \mathrm{~mm}$ de diâmetro e o segundo modelo com $1050 \mathrm{~mm}$ de comprimento e $200 \mathrm{~mm}$ de diâmetro. Com relação ao posicionamento das lâmpadas, o modelo com uma lâmpada apresenta um orifício de $2300 \mathrm{~mm}$ de comprimento por $300 \mathrm{~mm}$ de diâmetro. Já para o reator 2, onde utilizou-se quatro lâmpadas, o orifício apresenta $2050 \mathrm{~mm}$ de comprimento e $450 \mathrm{~mm}$ de diâmetro. A figura 1 mostra a representação $3 \mathrm{D}$ dos modelos construídos no trabalha desenvolvido.

Após os testes, o autor pode afirmar que o reator 2 foi até $30 \%$ mais eficiente na remoção de fármacos em relação ao reator 1 .

Trabalhos usando processos com Fenton e foto-Fenton para tratamento de efluentes contaminados com diversos poluentes tais como antibióticos e pesticidas foram largamente relatados. Conforme Aziz, Asaithambi e Daud (2016), Lutterbeck et al. (2015), Sirés et al. (2007) e De la Cruz et al. (2013), este é um dos processos oxidativos mais utilizados e com alto potencial de oxidação para esses compostos. Como retratado no trabalho de Lutterbeck, foi 
utilizado uma lâmpada de mercúrio de média pressão com uma potência entre $500-700 \mathrm{~W}$ e as amostras permaneceram sobre irradiação UV de 02 a 256 minutos. Lutterberck realizou seu trabalho utilizando 16 amostras diferentes, variando a concentração de íons $2+i F e^{\square}$ entre 60,90 e $120 \mathrm{mg} / \mathrm{L}$ e de $\mathrm{H}_{2} \mathrm{O}_{2}$ entre 300,450 e $600 \mathrm{mg} / \mathrm{L}$, sendo que na concentração de 90 mg $2+\mathrm{Fe}^{-} / \mathrm{L}$ e $450 \mathrm{mg}$ de $\mathrm{H}_{2} \mathrm{O}_{2} / \mathrm{L}$ houve uma remoção de carbono orgânico total (TOC) de $74,7 \%$ e uma degradação de $100 \%$ do fármaco alvo em até 4 minutos.

De Boni e Camana (2014), utilizaram o processo de Fenton combinado com a eletrofloculação para a remoção de corantes da água do efluente do ensaio de líquido penetrante. Em um vaso reacional de $5 \mathrm{~m}^{3}$ de capacidade, ajustou-se $\mathrm{opH}$ em faixa ácida e adicionou-se $200 \mathrm{~g}$ de sulfato ferroso e 20 litros de $\mathrm{H}_{2} \mathrm{O}_{2}$. Após o procedimento descrito no artigo, avaliou-se que a água tratada apresentava uma melhora de $99,36 \%, 88,98 \%$ e $99,34 \%$ para Cor verdadeira, DQO e Turbidez, respectivamente.

Tomando como base o atual conceito de preservação ambiente, pode-se destacar como um meio de processo de oxidação uso de ozônio, uma vez que o composto é inofensivo ao meio ambiente. Andreozzi et al. (2005), Parsa e Negahdar (2012), Asghar, Raman e Daud (2015) e Richard et al. (2014), utilizaram-se do método de ozônio para tratar efluentes de estação de tratamento e remoção de produtos farmacêuticos de meio hídricos. Richard em seu trabalho utilizou $\mathrm{O}_{3}$ combinado com $\mathrm{H}_{2} \mathrm{O}_{2}$ para degradar diversos fármacos de suas amostras. Este valeuse de uma lâmpada de mercúrio de baixa pressão de $15 \mathrm{~W}$, uma concentração de $\mathrm{O}_{3}$ de 5 $\mathrm{mg} / \mathrm{L}$ e uma concentração de $\mathrm{H}_{2} \mathrm{O}_{2}$ de $1 \mathrm{~g} / \mathrm{L}$. As amostras foram mantidas sob irradiação por 90 minutos. O peróxido de hidrogênio residual foi neutralizado com a adição de $0,25 \mathrm{~mL}$ da enzima catalase.

Outro POA que vem chamando atenção do meio científico é o da foto catálise, pois este é um método com aceitável custo benefício. ServaGalvis et al. (2016), Hassan, Zhao e Xie (2016) e Giraldo-Aguirre et al. (2015) trataram suas amostras para remoção de antibióticos. Valendo do trabalho de Serva-Galvis, que utilizou uma lâmpada de luz UV de $30 \mathrm{~W}$ de potência e condicionou suas amostras com $1000 \mu \mathrm{M}$ de $\mathrm{H}_{2} \mathrm{O}_{2}$ e $0,5 \mathrm{~g} / \mathrm{L}$ de $\mathrm{TiO}_{2}$. As amostras foram então irradiadas por cerca de 45 minutos, e após esse tempo, foi atingido uma taxa de remoção de aproximadamente $90 \%$ para os fármacos utilizados.

Jerônimo et al. (2012) utilizou, para a degradação de efluentes industriais oleoso, como o gerado pela castanha de caju, o procedimento de oxidação catalítica com o catalisador K$\mathrm{MnO}_{2} / \mathrm{CeO}_{2}$. Avaliando variáveis como: concentração mássica do catalisador $(4,0$ e 5,0/L) g/L, pressão total do sistema (300 e 900 psi) e temperatura $\left(80\right.$ a $\left.130{ }^{\circ} \mathrm{C}\right)$, os ensaios evidenciaram que o procedimento atingiu níveis de eficiência superiores a $90 \%$.

Considerando que há diversos tipos de POAs, estes podem variar quanto a sua utilização e aplicação. Visando a degradação de determinado tipo de compostos, deve se elencar qual processo oxidativo avançado é o ideal, considerando a viabilidade e o custo-benefício. Sabe-se que para cada processo há alteração em algumas características, tais como: concentração de $\mathrm{H}_{2} \mathrm{O}_{2}$, modelo e potência de lâmpada, tempo de radiação ou reação, concentração de ferro (quando utilizado) e entre outros. Segue abaixo a Tabela 2 contendo diversos trabalhos de POAs e materiais utilizados.

Estudos sobre a relação custo-benefício de POAs já foram realizados por alguns autores. No trabalho escrito por Bolton et al. 2003 realizou-se análises de diversas variáveis quanto a viabilidade dos processos, avaliando principalmente o consumo de energia elétrica. Bolton constatou que um processo utilizando cerca de $3.0 \mathrm{kWh} / \mathrm{m}^{3}$, com o custo de 0,08 USD/kWh o custo final será de aproximadamente $0,72 \mathrm{USD} / \mathrm{m}^{3}$ de água tratada, sem considerar o custo de químicos, operação entre outros. Frente a isso, torna-se necessário o estudo aprofundado das características do material tratado para possibilitar uma maior redução do custo final.

Dentre as classes de micropoluentes pode-se destacar os fármacos. Após o consumo de medicamentos, um determinado percentual não é absorvido pelo organismo, sendo eliminado, por exemplo, através da urina, com sua estrutura molecular e características biológicas específicas ainda ativas. Devido ao descarte e tratamento inapropriado (ou inexistente) desses resíduos, essa é uma porta de entrada para a contaminação de arroios, lagos e rios. Como consequência, estudos apontam que, mesmo em baixas concentrações, esses compostos podem causar efeitos adversos aos organismos aquáticos expostos à esse 
ambiente. Alguns exemplos são: a desregulação endócrina, alterando até mesmo a reprodução de peixes, e no caso de humanos, especificamente quanto ao uso de antibióticos, podem ocasionar em resistência bacteriana. Tais compostos sintéticos, devido sua alta complexidade, podem acabar não sendo removidos por sistemas de tratamentos convencionais, o que torna importante o estudo sobre o espectro de ação e a características de tais compostos, assim como outros meios de remoção destes micropoluentes (Bila e Dezzoti 2003; Liu et al. 2012; Mondragón et al. 2011; Isidori, Bellotta e Parrella 2009).

A Amoxicilina, assim como a Cefalexina, são antibióticos do grupo dos $\beta$-lactâmicos, usados largamente na indústria farmacêutica para o combate de bactérias. Devido a sua estrutura complexa, tais fármacos podem se tornar um problema em contato com as águas de rios e lagos, pois os tratamentos convencionais não são eficazes para sua degradação, fazendo com que estes cheguem inalterados até 0 consumidor.

Frente a estas dificuldades, os POAs estão sendo otimizados para a degradação de compostos complexos como a amoxicilina e a cefalexina. Segundo a literatura, diversos tipos de POAs podem ser aplicados, contudo, os mais utilizados são o de Fenton e $\mathrm{UV} / \mathrm{H}_{2} \mathrm{O}_{2}$. Em tais métodos, a amoxicilina pode alcançar taxas de 99\% de degradação, assim como uma degradação de aproximadamente $50 \%$ no carbono orgânico total.

Focalizando-se no fármaco Cefalexina, um dos melhores métodos para sua eficaz remoção é utilizando-se de luz ultravioleta (UV). Collado et al. (2014), valeu-se em seu trabalho de uma lâmpada UV de média pressão com irradiação de $40 \mathrm{~mJ} / \mathrm{cm}^{2}$ para tratar $50 \mathrm{~mL}$ de efluentes de uma estação de tratamento convencional de água e abastecimento. Após 3 e 9 horas de exposição a luz UV foi obtido uma taxa entre $35 \%$ e $70 \%$ de remoção do fármaco.

Alguns reatores têm sido desenvolvidos para tratamentos de água e efluentes, como visto na patente de Qiaoliang et al. (2016), onde estes desenvolveram uma torre para tratamento de água residual através de POAs e na patente de Ross et al. (2016) que usou-se um reator de oxidação fotocatalítica com emissão de radiação UV nos comprimentos de onda entre $100 \mathrm{~nm}$ até $400 \mathrm{~nm}$ com o intuito de tratar águas de abastecimento.

Ocanã (2013), patenteou um procedimento utilizando a combinação de ozônio com $\mathrm{H}_{2} \mathrm{O}_{2}$ para purificar óleos vegetais. $\mathrm{O}$ azeite produzido apresenta maior qualidade, acidez suave e odor agradável.

\section{CONCLUSÃO}

De acordo com o referencial teórico levantado, pode-se verificar que os POAs são tratamentos eficazes para compostos difíceis de serem degradados. Contudo, deve-se elencar o processo mais eficiente para o objeto de estudo, pois existem diversos tipos de POAs, sendo que alguns não são economicamente viáveis, prejudiciais ao meio ambiente ou simplesmente ineficaz contra um composto específico. Atualmente os POAs que mais tem aparecido em pesquisa são o de Fenton e o UV/ $\mathrm{H}_{2} \mathrm{O}_{2}$.

Tais processos ainda são relativamente novos na comunidade científica, sendo assim, torna-se necessário aprimorar as técnicas de oxidação avançada para se tornar viável aplicálas na prática em larga escala. Para quantificar a presença e a degradação de poluentes tem se tornado comum a análise por cromatografia a líquido acoplada a espectrometria de massas devido a sua grande precisão e sensibilidade.

\section{REFERÊNCIAS}

1. Alvárez, P. M.; García-Araya, J. F.; Beltrán F. J.; Giráldez, I.; Jaramillo J.; Gómez-Serrano, V. Carbon, 2006, 3102-3112.

2. Andreozzi, Roberto; Canterino, Marisa, Marotta, Raffaele; Paxeus, Nicklas. Journal Of Hazardous Materials, 2005, 243-250.

3. Asghar, Anam; Raman, Abdul A. A.; Daud, Wan M. A. W. Journal Of Cleaner Production, 2015, 826-838.

4. Audenaert, W. T. M.; Vandierendonck, D.; Van Hulle, S. W. H.; Nopens, I. Water Research, 2013, 2387-2398.

5. Autin, Olivier; Hart, Julie; Jarvis, Peter; Macadam, Jitka; Parsons, Simon A.; Jefferson, Bruce. Water Research, 2013, 2041-2049.

6. Ay, Filiz; Kargi, Fikret. Journal Of Hazardous Materials, 2010, 622-627.

7. Ayoub, Kaidar; Hullebusch, Eric D.; Cassir, Michel; Bermond, Alain. Journal of Hazardous Materials, 2010, 10-28.

8. Aziz, Abdul R. A.; Asaithambi, P.; Daud, Wan M. A. B. W. Process Safety And 
Environmental Protection, 2016, 227-235.

9. Benotti, Mark J.; Stanford, Benjamin D.; Wert, Eric C.; Snyder, Shane A. Water Research, 2009, 1513-1522.

10. Bila, Daniele M.; Dezotti, Márcia. Química Nova, 2003, 523-530.

11. Bila, Daniele M.; Dezotti, Márcia. Química Nova, 2007, V. 30, 651-666.

12. Bolong, Nurmin, Ismail, Ahmad F., Salim, Mohd R., Matsuura, T. Desalination, 2009, 229-246.

13. Bolton, James R.; Bircher, Keith G.; Tumas, William; Tolman, Chadwick A. Pure and Applied Chemistry, 2001, 627-637.

14. Chen, Wen S.; Juan, Chien N.; Wei, Kuo M. Chemosphere, 2005, 1072-1079.

15. Cheng, Min; Zeng, Guangming; Huang, Danlian; Lai, Cui; Xu, Piao; Zhang, Chen; Liu, Yang. Chemical Engineering Journal, 2016, 582-598.

16. Collado, N.; Rodriguez-Mozaz, S.; Gros, M.; Rubirola, A.; Barceló, D.; Comas, J.; Rodriguez-Roda, I.; Buttiglieri, Environmental Pollution, 2014, 202-212.

17. Comninellis, Christos; Kapalka, Agnieszka; Malato, Sixto; Parsons, Simon A.; Poulios, Ioannis; Mantzavinos, Dionissios. J Chem Technol Biotechnol, 2008, 769-776.

18. De Boni, Luis A. B.; Camana, Jackson. Tche Química, 2014, 79-84.

19. De La Cruz, N.; Esquius, L.; Grandjean, D.; Magnet, A.; Tungler, A.; Alencastro, L. F.; Pulgarín, C. Water Research, 2013, 58365845.

20. Elmolla, Emad S.; Chaudhuri, Malay. Desalination, 2010, 43-47.

21. Fenoll, José; Sabater, Paula; Navarro, Gines; Pérez-Lucas, Gabriel; Navarro, Simón. Journal of Hazardous Materials, 2013, 370379.

22. Fokin, Andrey A.; Schreiner, Peter R. Chemical Reviews, 2002, 1551-1593.

23. Ganiyu, Soliu O.; Van Hullebusch, Eric D.; Cretin, Marc; Esposito, Giovanni; Oturan, Mehmet A. Separation And Purification Technology, 2015, 891-914.

24. Giannakis, Stefanos; Vives, Franco A. G.; Grandjean, Dominique; Magnet, Anoys; Alencastro, Luiz F.; Pulgarin, César. Water Research, 2015, 295-306.

25. Giraldo-Aguirre, Ana L.; Erazo-Erazo, Edgar D.; Flórez-Acosta, Oscar A.; Serna-Galvis, Efraim A.; Torres-Palma, Ricardo A. Journal Of Photochemistry And Photobiology A:
Chemistry, 2015, 95-103.

26. Gros, Meritxell, Petrovic, Mira, Barcelo' Damià. In: Water Pollution, 2008, 37-104.

27. Hansen, Kamilla M. S.; Spiliotopoulou, Aikaterini; Chhetri, Ravi K.; Casas, Mònica E.; Bester, Kai; Andersen, Henrik R. Chemical Engineering Journal, 2016, 507-514.

28. Hassan, Muhammad; Zhao, Yaping; Xie, Bing. Chemical Engineering Journal, 2016, 264-275.

29. Homem, Vera; Alves, Arminda, Santos, Lúcia. Science of The Total Environmental, 2010, 6272-6280.

30. Hsueh, C. L.; Huang, Y. H.; Wang, C. C.; Chen, C. Y. Chemosphere, 2005, 1409-1414.

31. Ikehata, Keisuke; Naghashkar, Naeimeh J.; El-Din, Mohamed G. Ozone: Science And Engineering, 2006, 353-414.

32. Instituto Mexicano de la Propriedad Industrial (Culiacán, México), Ocanã, Guadalupe L. Ozonisation of vegetable oils by na advanced oxidation process (AOP), MX 2011012464 A, 08 nov. 2011, 14 mai. 2013.

33. Jerônimo, Carlos E. M.; Fernandes, Hermano G.; Sousa, João F. Tche Química, 2012, 1320.

34. Jo, Chang Hyun; Dietrich, Andrea M.; Tanko, James M. Water Research, 2011, 2507-2516.

35. Jung, Yeon J.; Kim, Wan G.; Yoon, Yeojoon; Kang, Joon-Wun; Hong, Young M.; Kim, Hyun W. Science Of Total Environmental, 2012, 160-167.

36. Katsoyiannis, Ioannis A.; Canonica, Silvio; Von Gunten, Urs. Water Research, 2011, 3811-3822.

37. Köhler, C.; Venditti, S.; Igos, E.; Klepiszewski, K.; Benetto, E.; Cornelissen, A. Journal Of Hazardous Materials, 2012, 70-77.

38. Lázár, K.; Calleja, G.; Melero, J. A.; Martinez, F.; Molina, R. Studies in Surface Science and Catalysis, 2004, 805-812.

39. Linden, Karl G.; Rosenfeldt, Erik J.; Chen, Pei J.; Kullman, Seth W. Water Science and Technology, 2007, 313-325.

40. Liu, Ning; Sijak, Sasa; Zheng, Ming; Tang, Liang; Xu, Gang; Wu, Minghong. Chemical Engineering Journal, 2015, 826-834.

41. Liu, Pengxiao; Zhang, Hanmin; Feng, Yujie; Yang, Fenglin; Zhang, Jianpeng. Hemical Engineering Journal, 2014, 211-220.

42. Liu, Xiaoshan; Lee, Jinyoung; Ji, Kyunghee; Takeda, Shunichi; Choi, Kyungho. Toxicology Letters, 2012, 70-76.

43. Lopez, Antonio; Bozzi, Anna; Mascolo, 
Giuseppe; Kiwi, John. Journal Of Photochemistry And Photobiology A: Chemistry, 2003, 121-126.

44. Lutterbeck, Carlos A.; Baginska, Ewelina; Machado, Ênio L.; Kümmerer, Klaus. Chemosphere, 2015, 290-296.

45. Lutterbeck, Carlos A.; Wilde, Marcelo L.; Baginska, Ewelina; Leder, Chrisroph; Machado, Ênio L.; Kümmerer, Klaus. Science Of The Total Environmental, 2015, 232-245.

46. Mondragón, Verónica A.; Llamas-Pérez, Dámaris F.; González-Guzmán, Gladis E.; Márquez-González, Antonio R.; PadillaNoriega, Roberto; Durán-Avelar, M. J.; Franco, Bernardo. Environ Monit Assess, 2011, 329-340.

47. Neyens, E.; Baeyens, J. Journal Of Hazardous Materials, 2003, 33-50.

48. Ohtani, Bunsho. Phys. Chem. Chem. Phys., 2014, 1788-1797.

49. Pal, Amrita, Gin, Karina Y.-H., Lin, Angela Y.C., Reinhard, Martin. Science Of The Total Environment, 2010, 6062-6069.

50. Parrino, F.; Camera-Roda, G.; Loddo, V.; Augugliaro, V.; Palmisano L. Applied Catalysis B: Environmental, 2015, 37-43.

51. Parsa, Basiri J.; Nagahdar, Hagh S. Separation And Purification Technology, 2012, 315-320.

52. Pereira, Vanessa J.; Linden, Karl G.; Weinberg, Howard S. Water Research, 2007, 4413-4423.

53. Pourakbar, Mojtaba; Moussavi, Gholamreza; Shekoohiyan, Sakine. Ecotoxicology And Environmental Safety, 2016, 72-77.

54. Quan, Xie; Zhao, Xu; Chen, Shuo; Zhao, Huimin; Chen, Jingwen; Zhao, Yazhi. Chemosphere, 2005, 266-273.

55. Ribeiro, Ana R.; Nunes, Olga C.; Pereira, Manuel F. R.; Silva, Adrián M. T. Environment International, 2015, 33-51.

56. Richard, Jessica; Boergers, Andrea; Vom Eyser, Claudia; Bester, Kai; Tuerk, Jochen. International Journal Of Hygiene And Environmental Health, 2014, 506-514.

57. Rodrigues, Bárbara M.; Cerqueira, Alexandre A.; Russo, Carlos; Marques, Monica R. C. Tche Química, 2010, 7-15.

58. Rodríguez, S.M.; Gálvez, J. B.; Rubio, M. I. M.; Ibáñez, P. F.; Gernjak, W.; Alberola I. O. Chemosphere, 2005, 391-398.

59. Santos, Lucilaine V. S.; Meireles, Alexandre M.; Lange, Liséte C. Journal Of Environmental Management, 2015, 8-12.
60. Serna-Galvis, Efraim A.; Silva-Agredo, Javier; Giraldo, Ana L.; Flórez, Oscar A.; TorresPalma, Ricardo A. Chemical Engineering Journal, 2016, 953-962.

61. Shu, Zengquan; Bolton, James R.; Belosevic, Miodrag; El Din, Mohamed G. Water Research, 2013, 2881-2889.

62. Sirés, Ignasi; Garrido, José A.; Rodríguez, Rosa M.; Brillas, Enric; Oturan, Nihal; Oturan, Mehmet A. Applied Catalysis, 2007, 382-394.

63. Snyder, Shane A.; Wert, Eric C.; Lei, Hongxia; Westerhoff, Paul; Yoon, Yeomin. Removal of EDCs and Pharmaceuticals in Drinking and Reuse Treatment Processes. Denver, Awwa Research Foundation, 2007.

64. Snyder, Shane A.; Westerhoff, Paul; Yoon, Yeomin; Sedlak, David L. Environmental Engineering Science, 2003, 449-469.

65. State Intellectual Property Office of the P.R.C (China), Qiaoliang, Sun; Haiwei, Sun; Hailing, Sun; Hainuo, Sun. AOPs sewage treatment tower, CN 205204892 U, 25 nov. 2015, 24 fev. 2016.

66. Vilhunen, Sari; Vilve, Miia; Vepsäläinen, Mikko; Sillanpää, Mika. Journal of Hazardous Materials, 2010, 776-782.

67. Von Gunten, Urs. Water Research, 2003, 1443-1467.

68. Wang, Lei; Jiang, Xuanzhen; Liu, Yongjun. Journal of Hazardous Materials, 2008, 11061114.

69. Wols, B. A.; Harmsen, D. J. H.; Van Remmen, T.; Beerendonk, E. F.; Hofman-Caris; C. H. M. Chemical Engineering Science, 2015, 712721.

70. Wols, B. A.; Hofman-Caris, C. H. M. Water Research, 2012, 2815-2827.

71. World Intellectual Property Organization, Clarke, Ross. Apparatus and method for water treatment, WO 2016/020692 A2, 06 ago. 2015, 11 fev. 2016.

72. Yang, Yi; Pignatello, Joseph J.; Ma, Jun; Mitch, William A. Water Research, 2016, 192200.

73. Yuan, Fang; Hu, Chun; Hu, Xuexiang; Wei, Dongbin, Chen, Yong; Qu, Jiuhui. Journal Of Hazardous Materials, 2011, 1256-1263. 


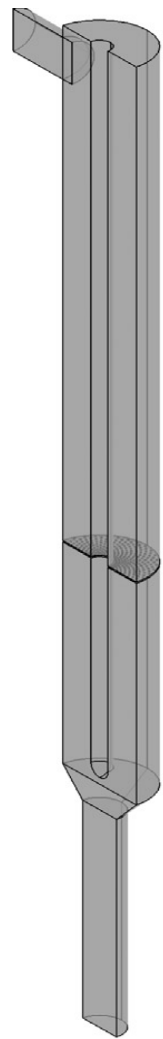

Reator 1

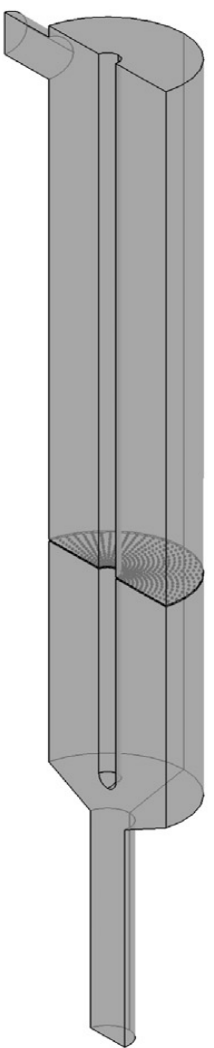

Reator 2

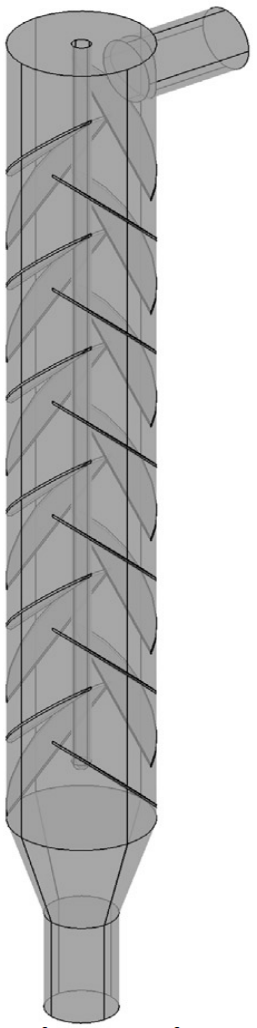

Mixador anular com 1 lâmpada

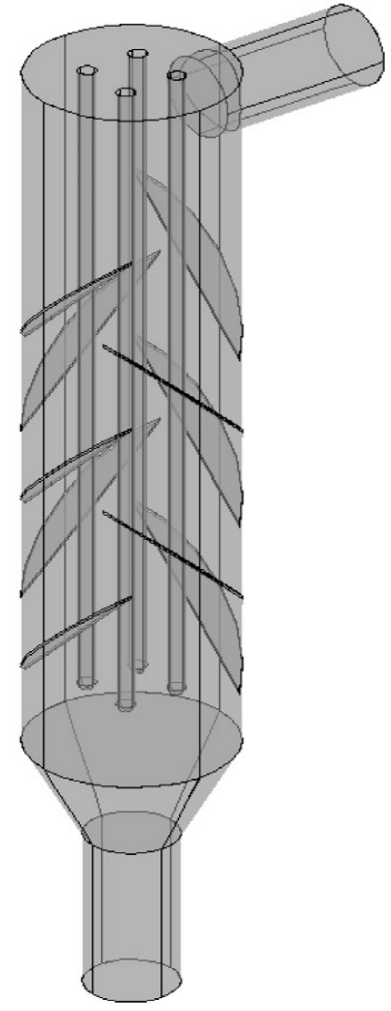

Mixador anular com 4 lâmpadas

Figura 1: Representação 3D dos reatores e mixadores desenvolvidos (Wols et al. 2015). 
Tabela 2: Relação de trabalhos envolvendo POAs e suas características.

\begin{tabular}{|c|c|c|c|c|c|}
\hline Amostra & $\begin{array}{c}\text { Tipo } \\
\text { de POA }\end{array}$ & Lâmpada/Potência & $\begin{array}{l}\text { Conc. de } \\
\text { Peróxido elou } \\
\text { Ferro }\end{array}$ & $\begin{array}{c}\text { Tempo de radiação } \\
\text { ou reação }\end{array}$ & Ref. \\
\hline Padrão de 5-FU & $\mathrm{UV} / \mathrm{H}_{2} \mathrm{O}_{2}$ & $\begin{array}{c}\text { Lâmpada de mercúrio de } \\
\text { média pressão TQ150 }\end{array}$ & $\begin{array}{c}9,8 ; 14,7 \\
e \\
19,6 \mathrm{mM}\end{array}$ & $\mid \begin{array}{c}2,4,8,16,32,64,128 \text { e } 256 \\
\min \end{array}$ & [45] \\
\hline Água residual & Fenton & $6 \mathrm{~W}, 365 \mathrm{~nm}$ & 100 até $300 \mathrm{mg} / \mathrm{L}$ & $50 \mathrm{~min}$ & [20] \\
\hline $\begin{array}{l}\text { Efluente de } \\
\text { estação de } \\
\text { tratamento }\end{array}$ & $\mathrm{UV} / \mathrm{H}_{2} \mathrm{O}_{2}$ & $\begin{array}{c}\text { Lâmpada de } \mathrm{Hg} \text { de média } \\
\text { pressão } \\
15 \mathrm{~W}, 254 \mathrm{~nm}\end{array}$ & $17-170 \mathrm{mg} / \mathrm{L}$ & $5 \mathrm{~min}$ & {$[72]$} \\
\hline Padrão & $\begin{array}{l}\text { Fenton sem } \\
\text { UV }\end{array}$ & -------- & $\begin{array}{c}\mathrm{H}_{2} \mathrm{O}_{2}: 3.50-4.28 \\
\mathrm{mg} / \mathrm{L} \\
\mathrm{Fe}: 254-350 \mu \mathrm{g} / \mathrm{L} \\
\end{array}$ & $30 \mathrm{~min}$ & [29] \\
\hline Padrões & $\mathrm{UV} / \mathrm{H}_{2} \mathrm{O}_{2}$ & $\begin{array}{c}\text { Lâmpada de } \mathrm{Hg} \text { de média } \\
\text { pressão } \\
1 \mathrm{~kW}\end{array}$ & 25 e $50 \mathrm{mg} / \mathrm{L}$ & Até $8 \mathrm{~min}$ & [61] \\
\hline Solução Padrão & UV & $\begin{array}{c}\text { Hg de baixa pressão } \\
5.7 \mathrm{~W} \\
254 \mathrm{~nm} \\
\end{array}$ & ---------- & $50 \mathrm{~min}$ & {$[53]$} \\
\hline $\begin{array}{l}\text { Padrões e } \\
\text { efluente de } \\
\text { estação de } \\
\text { tratamento }\end{array}$ & $\mathrm{UV} / \mathrm{H}_{2} \mathrm{O}_{2}$ & $\begin{array}{l}\text { Lâmpada de baixa } \\
\text { pressão de } 30 \mathrm{~W}\end{array}$ & $8 \mathrm{mM}$ & ---------- & [05] \\
\hline Água residual & $\mathrm{UV} / \mathrm{H}_{2} \mathrm{O}_{2}$ & $60 \mathrm{~W}$ & $\begin{array}{c}200 \mathrm{mg} / \mathrm{L} \text { até } 800 \\
\mathrm{mg} / \mathrm{L} \text { de peróxido } \\
30 \%\end{array}$ & ------- & [23] \\
\hline $\begin{array}{c}\text { Efluente de } \\
\text { estação de } \\
\text { tratamento e } \\
\text { água de rio }\end{array}$ & $\mathrm{UV} / \mathrm{H}_{2} \mathrm{O}_{2}$ & \begin{tabular}{|c|} 
Lâmpada de Hg de baixa \\
pressão de $15 \mathrm{~W}$ e de \\
média pressão de $500-$ \\
$700 \mathrm{~W}$ \\
\end{tabular} & $6,8 \mathrm{mg} / \mathrm{L}$ & ----- & [36] \\
\hline Solução aquosa & $\begin{array}{l}\text { Fenton sem } \\
\text { luz UV }\end{array}$ & 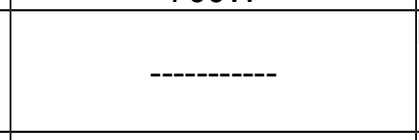 & $\begin{array}{l}\mathrm{H} 2 \mathrm{O} 2: 10-500 \mathrm{mg} / \mathrm{L} \\
\mathrm{Fe}(\mathrm{II}): \text { até } 50 \mathrm{mg} / \mathrm{L}\end{array}$ & $2,5 \mathrm{~min}$ & {$[06]$} \\
\hline $\begin{array}{l}\text { Efluente } \\
\text { Hospitalar }\end{array}$ & $\mathrm{UV} / \mathrm{H}_{2} \mathrm{O}_{2}$ & \begin{tabular}{|c|} 
Lâmpada de mercúrio de \\
baixa pressão de $0,25 \mathrm{~kW}$ \\
e de média pressão de $2-$ \\
$10 \mathrm{~kW}$
\end{tabular} & 0,56 e $1,11 \mathrm{~g} / \mathrm{L}$ & $\begin{array}{c}\text { 91 s para lâmpada de } \\
\text { média pressão e } 1013 \mathrm{~s} \\
\text { para lâmpada de baixa } \\
\text { pressão }\end{array}$ & {$[37]$} \\
\hline Padrão & $\mathrm{UV} / \mathrm{H}_{2} \mathrm{O}_{2}$ & $\begin{array}{l}\text { Mercúrio de baixa } \\
\text { pressão } \\
254 \mathrm{~nm} \\
\end{array}$ & $10 \mathrm{mM}$ de $\mathrm{H}_{2} \mathrm{O}_{2}$ & $80 \mathrm{~min}$ & [35] \\
\hline $\begin{array}{l}\text { Efluentes de } \\
\text { estação de } \\
\text { tratamento }\end{array}$ & $\mathrm{UV} / \mathrm{H}_{2} \mathrm{O}_{2}$ & $\begin{array}{l}\text { Lâmpada de baixa } \\
\text { pressão de } 11 \mathrm{~W}\end{array}$ & 2 e $4 \mathrm{mM}$ & $70 \mathrm{~min}$ & [04] \\
\hline Padrão & $\mathrm{UV} / \mathrm{H}_{2} \mathrm{O}_{2}$ & $\begin{array}{c}\text { Mercúrio de alta pressão } \\
300 \mathrm{~W} \\
\end{array}$ & Até $5 \mathrm{mM}$ & $120 \mathrm{~min}$ & {$[40]$} \\
\hline $\begin{array}{l}\text { Efluente de } \\
\text { estação de } \\
\text { tratamento }\end{array}$ & UV & -.. & \begin{tabular}{|c|} 
Lâmpada de média \\
pressão \\
$40 \mathrm{~mJ} / \mathrm{cm}^{2}$ \\
$254 \mathrm{~nm}$ \\
\end{tabular} & 3 e 9 horas & [16] \\
\hline
\end{tabular}

\section{FONTE: DOS AUTORES}

PERIÓDICO TCHÊ QUÍMICA • www.periodico.tchequimica.com • Vol. 14 N. 27. • ISSN 1806-0374 (impresso) • ISSN 1806-9827 (CD-ROM) • ISSN 2179-0302 (meio eletrônico) 